Annals of Pure and Applied Mathematics

Vol. 18, No. 2, 2018, 207-212

ISSN: 2279-087X (P), 2279-0888(online)

Published on 28 December 2018

www.researchmathsci.org

DOI: http://dx.doi.org/10.22457/apam.v18n2a11

Annals of

Pure and Applied

Mathematics

\title{
On Completion Problems for Various Subclasses of $P_{0}^{+}$-Matrices
}

\section{Victor Tomno}

Department of Mathematics and Physics, Moi University

P.O Box 3900-30100, Eldoret, Kenya. E-mail: victomno@ gmail.com

Received 12 November 2018; accepted 23 December 2018

Abstract. In this paper, we study completions for weakly sign symmetric $p_{0}^{+}$-matrices, sign symmetric $p_{0}^{+}$-matrices and nonnegative $p_{0}^{+}$-matrices. We obtained that digraphs that include all loops and have weakly sign symmetric $p_{0}^{+}$-completion, sign symmetric $p_{0}^{+}$-completion and nonnegative $p_{0}^{+}$-completion are complete digraphs.

Keywords: Matrix completion, partial matrix, digraphs, weakly sign symmetric $P_{0}^{+}$matrix, sign symmetric $P_{0}^{+}$-matrix, nonnegative $P_{0}^{+}$-matrix.

AMS Mathematics Subject Classification (2010): 15A48

\section{Introduction}

In this section we define terms and give a brief literature on related work.

Definition 1.1. A $P$-matrix ( $P_{0}$-matrix) is a matrix in which every principal minor of the matrix $A$ is positive (nonnegative) [1].

Definition 1.2. A $n \times n$ matrix is a $P_{0}^{+}$-matrix if for each $k \in\{1, \ldots, n\}$, every $k \times k$ principal minor is nonnegative and at least one $k \times k$ principal minor is positive [2].

Clearly, $P$-matrix is both $P_{0}$-matrix and $P_{0}^{+}$-matrix. Also observe that $P_{0}^{+}$-matrix is a $P_{0}$-matrix.

Definitions 1.1 and 1.2 considers the values of the principal minors, the next definition gives restrictions on the type of entries of a matrix.

Definition 1.3. A $n \times n$ matrix $A=\left\lfloor a_{i j}\right\rfloor$ is

i. Weakly sign symmetric(wss) if $a_{i j} a_{j i} \geq 0$ for all $i$ and $j$

ii. Sign symmetric(ss) if $a_{i j} a_{j i}>0$ or $a_{i j}=a_{j i}=0$ for all $i$ and $j$ 
Victor Tomno

iii. Nonnegative if $a_{i j} \geq 0$ for all $i$ and $j$

iv. Positive if $a_{i j}>0$ for all $i$ and $j$

Using Definition 1.3, we have four different subclasses of $P_{0}^{+}$-matrix (given in Definition 1.2).

Definition 1.4. A $P_{0}^{+}$-matrix A is called a weakly sign symmetric $P_{0}^{+}$-matrix (resp. sign symmetric $P_{0}^{+}$-matrix) if $a_{i j} a_{j i} \geq 0$ (resp. either $a_{i j} a_{j i}>0$ or $a_{i j}=0=a_{j i}$ ) for all $i$ and $j$. Similarly, A $P_{0}^{+}$-matrix $\mathrm{A}$ is called a positive $P_{0}^{+}$-matrix (resp. nonnegative $P_{0}^{+}$-matrix) if $a_{i j} a_{j i}>0$ (resp. $a_{i j} a_{j i} \geq 0$ ) for all $i$ and $j$.

Example 1.5. The matrix $A=\left[\begin{array}{ccc}6 & -2 & 2 \\ -3 & 4 & 1 \\ 0 & 2 & 2\end{array}\right]$ is a $P_{0}^{+}$-matrix since all principal minors are nonnegative and in every order there is at least one positive principal minor. Looking at the entries, it is clear that matrix $A$ is a weakly sign symmetric $P_{0}^{+}$-matrix. It fails to be sign symmetric $P_{0}^{+}$-matrix because $a_{13}=2 \neq a_{31}=0$, again it is not a nonnegative $P_{0}^{+}$matrix since both $a_{12}=-2$ and $a_{21}=-3$ are negatives and by the same fact it is not a positive $P_{0}^{+}$-matrix.

Definition 1.6. A $P_{0,1}^{+}$-matrix is a $P_{0}^{+}$-matrix whose diagonal entries positive and a positive $P_{0,1}^{+}$-matrix is a $P_{0,1}^{+}$-matrix in which all entries are positive.

Proposition 1.7. A matrix is a positive $P_{0,1}^{+}$-matrix if and only if it is positive $P_{0}^{+}$matrix

Proof: Positive $P_{0,1}^{+}$-matrix is a $P_{0,1}^{+}$-matrix in which all entries are positive (from Definition 1.6), it means the condition that all diagonal entries are positive and hence it is a positive $P_{0}^{+}$-matrix.

Conversely, a positive $P_{0}^{+}$-matrix is a $P_{0}^{+}$-matrix in which all entries are positive hence all diagonal entries are positive, therefore it correct to say it is a positive $P_{0,1}^{+}$matrix (although diagonal entries have been repeatedly been mentioned to be positive).

Definition 1.8. A partial matrix is a matrix in which some entries are specified while others are free to be chosen. Let $\Pi$ be a class of matrices (e.g. weakly sign 
On Completion Problems for Various Subclasses of $P_{0}^{+}$- Matrices

symmetric $P_{0}^{+}$-, sign symmetric $P_{0}^{+}$-, nonnegative $P_{0}^{+}$- and positive $P_{0}^{+}$-matrices) then a partial $\prod$-matrix is one whose specified entries satisfy the required properties of a $\Pi$ matrix.

Graph theoretic approach will be used in completing these partial matrices, and some definitions are given as follows.

Definition 1.9. A digraph $D=\left(V_{D}, E_{D}\right)$ is a graph $G$ with ordered pairs $(u, v)$ of vertices and arc where $u$ the initial vertex is and $v$ is the terminal vertex. The order of a digraph $D$ denoted $n$ is the number of vertices of $D$. A digraph is complete digraph if it includes all possible arcs between its vertices (also called clique) [3].

A $n \times n$ partial matrix $A$ is said to specify a digraph $D$ on vertices $\left\{v_{1}, \ldots, v_{n}\right\}$ if $\left(v_{i}, v_{j}\right)$ is an arc in $D$ if and only if the entry $a_{i j}$ of $A$ is specified.

Definition 1.10. A completion of a partial matrix is a specific choice of values for the unspecified entries. If we consider classes given in Definition 2.8, a digraph $D$ has $\Pi$ completion if any partial $\Pi$-matrix specifying $D$ can be completed to a $\Pi$-matrix.

On the related work, we just give a brief history of matrix completions close to our research class. Research on $P$-matrix completion was first studied by Johnson and Kroschel in [4] and later extended by DeAlba and Hogben in [5]. In 2003, a subclass of $P$-matrices: weakly sign symmetric $P$-matrices was studied in [6] and then in two subclasses: positive and nonnegative $P$-matrices were considered in [7]. Another class on $P_{0}$-matrices was investigated first by Choi and others in [1], and its subclasses, weakly sign symmetric $P_{0}$-matrices, nonnegative symmetric $P_{0}$-matrices and sign symmetric $P_{0}$-matrices were consider in the following papers [6], [8] and [9] respectively. In 2015, a new class of $P_{0}^{+}$-matrices was first introduced and classification of digraphs of up to order 4 having $P_{0}^{+}$-completion was done. It is in this class that we are interested in, and the subclasses to be discussed are weakly sign symmetric $P_{0}^{+}$-matrices, sign symmetric $P_{0}^{+}$-matrices and nonnegative $P_{0}^{+}$-matrices.

\section{Preliminaries}

In this section, we will present some basic results that will be useful in the next section.

If a partial wss $P_{0}^{+}$-matrices, ss $P_{0}^{+}$-matrices and nonnegative $P_{0}^{+}$-matrices omits all diagonal entries then it can be completed to wss $P_{0}^{+}$-matrices, ss $P_{0}^{+}$-matrices and nonnegative $P_{0}^{+}$-matrices by assigning sufficiently large values to unspecified diagonal entries. In this research we are interested in the situations where all diagonal entries are 


\section{Victor Tomno}

specified. Zeros along diagonal entries tend to make completion for the three subclasses difficult.

Consider $A=\left[\begin{array}{ll}x & 1 \\ 2 & 0\end{array}\right]$ which is a partial wss $P_{0}^{+}$-matrix, partial ss $P_{0}^{+}$-matrix and a partial nonnegative $P_{0}^{+}$-matrix specifying digraph in Fig. 2.1 and cannot be completed to a wss $P_{0}^{+}$-matrix, a ss $P_{0}^{+}$-matrix and a nonnegative $P_{0}^{+}$-matrix respectively since $\operatorname{det} A=-2<0$ for any value of $x$. Thus the digraph in Figure 2.1 does not have wss $P_{0}^{+}$completion, ss $P_{0}^{+}$- completion and nonnegative $P_{0}^{+}$- completion

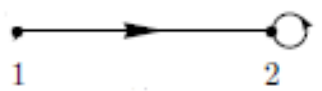

Figure 2.1:

Now, in the next section we assume that all digraphs have diagonal entries specified.

\section{Main results}

Our main results on completions of various subclasses of $P_{0}^{+}$- matrices namely weakly sign symmetric $P_{0}^{+}$- matrices, sign symmetric $P_{0}^{+}$-matrices and nonnegative $P_{0}^{+}$completion are presented in Theorem 3.1, 3.2 and 3.3 respectively.

Theorem 3.1. The digraphs having all loops and weakly sign symmetric $P_{0}^{+}$-completion are complete digraph.

Proof: Let wss $n \times n P_{0}^{+}$-matrix $A_{c}$ be a completion of partial wss $n \times n P_{0}^{+}$-matrix $A$ having all diagonal entries specified. Assume that the partial wss $n \times n P_{0}^{+}$-matrix $A$ has the first $n-1$ diagonal entries as 0 and the last is 1 with specified entries $a_{i j}{ }^{\prime} s$ and unspecified entries $x_{i j}{ }^{\prime} s$. Consider the $2 \times 2$ principal minors $\operatorname{det} A(i, j)$ for some $i, j \in\{1, \ldots, n\}$. Note that $d_{i} d_{j}=0$ always. Now split into three cases:

Case 1: Position $i j$ and $j i$ are specified. In this case we have

$$
\operatorname{det} A(i, j)=d_{i} d_{j}-a_{i j} a_{j i}=-a_{i j} a_{j i} \geq 0
$$

Thus $a_{i j} a_{j i} \leq 0$ and by wss $P_{0}^{+}$-completion $\left(a_{i j} a_{j i} \geq 0\right)$ we have $a_{i j} a_{j i}=0$

Case 2: Position $i j$ is specified and $j i$ is unspecified. In this case we have

$$
\operatorname{det} A(i, j)=d_{i} d_{j}-a_{i j} x_{j i}=-a_{i j} x_{j i} \geq 0
$$


On Completion Problems for Various Subclasses of $P_{0}^{+}$- Matrices

Thus $a_{i j} x_{j i} \leq 0$ and by wss $P_{0}^{+}$-completion we have $a_{i j} x_{j i}=0$

Case 3: Position $i j$ and $j i$ are unspecified. In this case we have

$$
\operatorname{det} A(i, j)=d_{i} d_{j}-x_{i j} x_{j i}=-x_{i j} x_{j i} \geq 0
$$

Thus $x_{i j} x_{j i} \leq 0$ and by wss $P_{0}^{+}$-completion we have $x_{i j} x_{j i}=0$

Observe that in all cases the product of twin entries is zero. However wss $P_{0}^{+}$completion requires that at least one of $2 \times 2$ principal minors is positive. This is a contradiction.

Theorem 3.2. The digraphs having all loops and sign symmetric $P_{0}^{+}$-completion are complete digraphs.

Proof: Using same hypothesis as in Theorem 3.1, again consider the $2 \times 2$ principal minor $\operatorname{det} A(i, j)$ for some $i, j \in\{1, \ldots, n\}$ and that $d_{i} d_{j}=0$ always. This means if a nondiagonal entry is specified then it must be zero (0) that is $a_{i j}=0$ since $\operatorname{det} A(i, j)=d_{i} d_{j}-a_{i j} x_{j i}=-x_{i j} a_{j i}<0$ if $a_{i j} \neq 0$. Therefore all unspecified nondiagonal twin entries $x_{j i}$ are also assigned zero (0) that is $c_{j i}=0$. As a result all nondiagonal entries have zeros hence $\operatorname{det} A(\alpha)=0 \forall \alpha \in\{1, \ldots, n\}$ this shows that partial ss $P_{0}^{+}$-matrices with unspecified entries lack sign symmetric $P_{0}^{+}$-completion and so, the only digraphs having all loops and sign symmetric $P_{0}^{+}$-completion are complete digraphs.

Theorem 3.3. The digraphs having all loops and nonnegative $P_{0}^{+}$-completion are complete digraph.

Proof: The proof for this theorem follows from the proof of Theorem 3.1, also having three cases with all specified entries $a_{i j}$ s being nonnegative i.e. $a_{i j} \geq 0$ and values assigned to unspecified entries $x_{i j}$ s being nonnegative that is $c_{i j} \geq 0$, which also shows that all the three cases have the product of the twin entries being zero and similar to Theorem 3.1 does not have $2 \times 2$ principal sub-matrix with positive determinant hence digraphs having all loops and nonnegative $P_{0}^{+}$-completion are complete digraph.

\section{Conclusion and recommendations}

Based on the main results we have concluded that digraphs that include all loops and have weakly sign symmetric $P_{0}^{+}$-completion, sign symmetric $P_{0}^{+}$-completion and nonnegative $P_{0}^{+}$-completion are complete digraphs. According to sections on related 


\section{Victor Tomno}

work and main results, we observe that similar research should be done for positive $P_{0}^{+}$matrices.

\section{REFERENCES}

1. J.Y.Choi, L.M.Dealba, L.Hogben and M.Maxwell, The $P_{0}$-Matrix Completion Problem, Electronic Journal of Linear Algebra, 9 (2002) 1-20.

2. B.K.Sarma and K.Sinha, The $P_{0}^{+}$-Matrix Completion Problem, Electronic Journal of Linear Algebra, 29 (2015) 120-143.

3. L.Hogben, Graph Theoretic Methods of Matrix Completion Problem, Linear Algebra and its Applications, 328 (2001) 161-202.

4. C.R.Johnson and B.K.Kroschel, The combinatorially symmetric $P$-matrix Completion Problem, Electronic Journal of Linear Algebra, 1 (1996) 59-64.

5. L.M.DeAlba, and L.Hogben, The completions $P$-matrix patterns, Linear Algebra and Application, 319(2000) 83-102.

6. L.M.DeAlba, T.L.Hardy, L.Hogben and A.Wangsness, The (Weakly) Sign Symmetric $P$-matrix Completion Problems, Electronic Journal of Linear Algebra, 10 (2003) 257-271.

7. J.Bowers, J.Evers, L.Hogben, S.Shaner, K.Snider and A.Wangsness, On completion problems for various classes of $P$-matrices, Linear Algebra and Application, 413 (2006) 342-354.

8. J.Y.Choi, L.M.DeAlba, L.Hogben, B.M.Kivunge, S.K.Nordstrom and M.Shedenhelm, The nonnegative $P_{0}$-matrix completion problem, Electronic Journal of Linear Algebra, 10 (2003) 46-59.

9. V.Tomno, The sign symmetric $P_{0}$-matrix completion problem, International Journal of Mathematical Archive, 9(11) (2018) 16-19

10. F.Harary, Graph Theory; New York; Addison-Wesley Publishing Company, 1969. 